[9] S. Kar, J. M. F. Moura, and K. Ramanan, "Distributed parameter estimation in sensor networks: Nonlinear observation models and imperfect communication,” Aug. 2008 [Online]. Available: http://arxiv.org/ abs/0809.0009

[10] S. Kar and J. M. F. Moura, "Distributed consensus algorithms in sensor networks with imperfect communication: Link failures and channel noise," IEEE Trans. Signal Process., vol. 57, no. 1, pp. 355-369, Jan. 2009.

[11] H. M. Rodrigues and J. Sola-Morales, "A note on the relationship between spectral radius and norms of bounded linear operators," Cadernos de Matematica, vol. 9, pp. 61-65, May 2008.

\section{Performance Analysis of Reconstruction Techniques for Frequency-Domain Optical-Coherence Tomography}

Chandra Sekhar Seelamantula and Michael Unser, Fellow, IEEE

Abstract-We address the issue of noise robustness of reconstruction techniques for frequency-domain optical-coherence tomography (FDOCT). We consider three reconstruction techniques: Fourier, iterative phase recovery, and cepstral techniques. We characterize the reconstructions in terms of their statistical bias and variance and obtain approximate analytical expressions under the assumption of small noise. We also perform Monte Carlo analyses and show that the experimental results are in agreement with the theoretical predictions. It turns out that the iterative and cepstral techniques yield reconstructions with a smaller bias than the Fourier method. The three techniques, however, have identical variance profiles, and their consistency increases linearly as a function of the signal-to-noise ratio.

Index Terms-Bias, cepstrum, frequency-domain optical-coherence tomography (FDOCT), variance.

\section{INTRODUCTION}

Frequency-domain optical-coherence tomography (FDOCT) [1] is a new imaging modality based on the principle of low-coherence interferometry [2]-[6]. It is contact-free, employs a nonionizing radiation, has high sensitivity, and enables fast acquisition. These advantages have given rise to many applications, the most recent ones being in functional imaging [7]-[9].

In FDOCT, the measurements are spectral data, from which the spatial domain description of the specimen must be computed. There are three state-of-the-art techniques for achieving this goal: 1) Fourier approach; 2) Iterative phase recovery method [10]; and 3) Cepstral technique [11].

Manuscript received February 03, 2009; accepted October 15, 2009. First published November 20, 2009; current version published February 10, 2010. The associate editor coordinating the review of this manuscript and approving it for publication was Dr. Maria Hansson-Sandsten. This work was supported by the Center for Biomedical Imaging of the Geneva-Lausanne universities and the EPFL, and the Hasler, Leenards and Louis-Jeantet foundations. A preliminary version of this work was presented at the International Conference on Acoustics, Speech, and Signal Processing 2008.

C. S. Seelamantula is with the Department of Electrical Engineering, Indian Institute of Science, Bangalore 560100, India (e-mail: chandra.sekhar@ieee. org).

M. Unser is with the Biomedical Imaging Group, École Polytechnique Fédérale de Lausanne, Lausanne 1015, Switzerland (e-mail: michael.unser@epfl.ch).

Color versions of one or more of the figures in this paper are available online at http://ieeexplore.ieee.org.

Digital Object Identifier 10.1109/TSP.2009.2037077

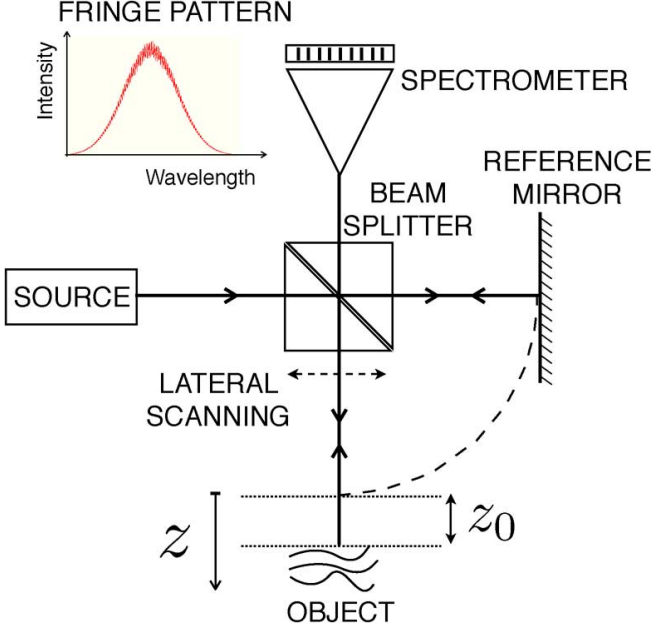

Fig. 1. Schematic of the FDOCT setup.

In practice, the measurements are affected by acquisition noise [15] that may affect the performance of a reconstruction algorithm. The contribution of this paper is a statistical characterization of the techniques mentioned above in the presence of noise in terms of the bias and variance of the reconstruction. These parameters are important because the bias is directly related to the amount of artifacts present in the FDOCT reconstruction, and the variance is a measure of the consistency of a reconstruction technique in the presence of experimental noise. We show that the bias-variance analysis is relatively easy for the Fourier approach. For the iterative and cepstral techniques, however, it is complicated because of the nonlinearities involved, and successive refinement of phase in the former. However, by making a small-noise assumption together with reasonable approximations, closed-form expressions can be derived. By means of Monte Carlo analyses, we show that the simulation results are largely in agreement with the theoretical analysis.

\section{SIGNAl ACQUiSITION AND MODEL}

The standard Michelson interferometric setup for FDOCT is shown in Fig. 1. The system comprises two arms, one for the object and another for the reference. A broadband light source (e.g., a super-luminescent diode) is employed for illumination as it yields high axial resolution. A beam-splitter is employed to divide the source output between the two arms. A mirror in the reference arm gives rise to the light that interferes with the light reflected from the object. The coherent interference of the recombined signals is detected by a spectrometer, which records the fringe intensity as a function of the wavelength $\lambda$. Equispaced samples on the wavenumber scale $k=2 \pi / \lambda$ are then obtained by interpolation. In the standard Fourier notation, the measured signal is expressed as $I(\omega)=S(\omega)|1+A(\omega)|^{2}$, where $A(\omega)$ is the Fourier transform of the backscattering function $a(z)$. We assume that $a(z)$ is causal, which is indeed the case in many standard configurations. The function $S(\omega)$ is the power spectrum of the source; $\omega=-2 k n(z)$, where $n(z)$ is the refractive index of the specimen as a function of the axial variable $z . S(\omega)$ is measurable, and hence known. The tomogram reconstruction problem is essentially the task of retrieving $a(z)$ given $I(\omega)$ and $S(\omega)$. An important point is that the light reflected from the object is much weaker than that reflected from the mirror; i.e., $|A(\omega)| \ll 1$. Since $n(z)$ is usually not known a priori, a standard simplification is obtained by replacing it with the average refractive index $\bar{n}$ [12, Ch. 12]. The tomograms would then be a function of the 
optical path length in the specimen. Such tomograms are quite standard in OCT applications.

\section{STATE-OF-THE-ART RECONSTRUCTION TECHNiQUES}

\section{A. Fourier Approach}

In this approach, the tomogram is reconstructed as: $\mathcal{F}^{-1}\{I\}(\omega)=$ $\left(r_{s s} * a\right)(z)+\left(r_{s s} * a\right)(-z)+\left(r_{s s} * r_{a a}\right)(z)$, where $r_{s s}(z)$ and $r_{a a}(z)$ are the autocorrelations of the source and $a(z)$, respectively; $\mathcal{F}$ denotes the Fourier transform operator. For broadband illumination, it is reasonable to assume that $r_{s s}(z)$ is a Dirac impulse. If the zero-delay plane is outside the specimen, or, equivalently, if $a(z)$ is causal, then there is no overlap between $\left(r_{s s} * a\right)(z)$ and $\left(r_{s s} * a\right)(-z)$. The autocorrelation $r_{a a}(z)$, however, is symmetric about $z=0$ and overlaps with $\left(r_{s s} * a\right)(z)$ and $\left(r_{s s} * a\right)(-z)$. Therefore, the distortion is $r_{a a}(z)$ and is additive.

\section{B. Iterative Phase Recovery Method}

This method is based on a Fienup technique for phase retrieval [14]. One starts with the spectral measurements and alternates between the spatial and spectral domains via the Fourier transform. At each iteration, two conditions are imposed: 1) causality in the spatial domain; and 2) the condition that the magnitude spectrum of the estimated scattering function matches the measurements. The procedure converges under some technical conditions [13]. After convergence, a minimum-phase version of the scattering function is obtained. If the scattering function is indeed minimum-phase, then, in principle, it can be recovered exactly [10], [13]. In [10], the authors also propose an analytical counterpart of the technique, which can be used for initialization to improve both accuracy and speed of convergence. A summary of the method is given below.

Initialization: $i=1 ; \phi^{(1)}(\omega)$ as per [10].

1. $H^{(i)}(\omega)=\sqrt{I(\omega)} e^{\mathrm{j} \phi^{(i)}(\omega)}$.

2. Inverse Fourier transform: $h^{(i)}(z)=\mathcal{F}^{-1}\left\{H^{(i)}\right\}(z)$.

3. Causality constraint: $a^{(i)}(z)=h^{(i)}(z) u(z)$, where $u(z)$ is the unit step function.

4. Fourier transform: $A^{(i)}(\omega)=\mathcal{F}\left\{a^{(i)}\right\}(\omega) ; \phi^{(i+1)}(\omega)=$ $\angle A^{(i)}(\omega)$.

5. Spectrum constraint: $H^{(i+1)}(\omega)=\sqrt{I(\omega)} e^{\mathrm{j} \phi^{(i+1)}(\omega)}$.

6. If a suitable criterion is met, $a^{(i)}(z)$ is the estimate of $a(z)$; else $i \leftarrow i+1$, and go to step 1 . The stopping criterion can be either a bound on $i$ or the relative difference between two consecutive estimates of $a(z)$.

\section{Cepstral Technique}

In this technique, the tomogram is reconstructed from the cepstrum instead of the spectrum. The advantage is that the the autocorrelation can be suppressed under realistic conditions ([11] contains the proof). The steps in the technique are given below.

1) Cepstrum: $c(z)=\mathcal{F}^{-1}\{\log (I / S)\}(z)$, where $I(\omega)$ and $S(\omega)$ are the measurements.

2) Causal cepstrum: $c^{+}(z)=c(z) u(z)$.

3) Fourier transformation: $C^{+}(\omega)=\mathcal{F}\left\{c^{+}\right\}(\omega)$.

4) Exponential mapping: $A(\omega)=\exp \left(C^{+}(\omega)\right)-1$.

5) Inverse Fourier transformation: $a(z)=\mathcal{F}^{-1}\{A\}(z)$.

\section{BIAS AND VARIANCE CALCUlations}

In order to formulate a realistic model for the noisy signal, we recall some results related to scattering and experimental studies on the noise characteristics. The measured signal is essentially due to scattering within the sample. Yaqoob et al. [16] have shown that optical scattering in static specimens, which may appear as stochastic, is in fact deterministic. The stochastic component is mainly due to the acquisition devices. Leitgeb et al. [15] have shown that the acquisition noise is approximately white. Based on these results, we employ the model of a deterministic signal in white noise. To conform to the practical scenario, we consider the samples of $a(z), A(\omega), S(\omega)$, and $I(\omega)$, denoted by $a_{k}, A_{k}, S_{k}$, and $I_{k}$, respectively. The finite-length discrete version of the measurements is therefore given as: $I_{k}=S_{k}\left|1+A_{k}\right|^{2}+W_{k}$, $0 \leq k \leq N-1$, where $\left|A_{k}\right| \ll 1$. $W_{k}$ is the measurement noise and is assumed to be Gaussian distributed with zero mean and variance $\sigma_{w}^{2}$. The Gaussian assumption has been shown to be sufficiently accurate for most practical applications. Based on this assumption, Ralston et al. [17] showed successful denoising results on experimental data. Also, in practice, the noise floor is about $40 \mathrm{~dB}$ below the signal level (small-noise regime) [15], [18], [19]; i.e., $\left|W_{k}\right| \ll S_{k}\left|1+A_{k}\right|^{2}$. We shall take advantage of this inequality in the subsequent analysis to derive approximate second-order expressions.

\section{A. Fourier Approach}

In this approach, it is a standard practice to do reconstruction after subtracting the reference signal (commonly known as background subtraction) because it enhances the sample structure. The measurements after this operation are $Q_{k}=$ $S_{k}\left|1+A_{k}\right|^{2}+W_{k}-S_{k}=2 S_{k} \operatorname{Real}\left\{A_{k}\right\}+S_{k}\left|A_{k}\right|^{2}+W_{k}$, $0 \leq k \leq N-1$. Taking the inverse discrete Fourier transform (IDFT) of the sequence ${ }^{1}\left\{Q_{k}\right\}_{0}^{N-1}$ and retaining the first half (the causal part), we get that $\widehat{a}_{m}=\left(r_{s s} * a\right)_{m}+\left(r_{s s} * r_{a a}\right)_{m}+w_{m}$, where $w_{m}$ is the noise in the spatial domain. Therefore, we have that $\mathcal{E}\left\{\widehat{a}_{m}\right\}=\left(r_{s s} * a\right)_{m}+\left(r_{s s} * r_{a a}\right)_{m}$, which indicates that $\widehat{a}_{m}$ is biased. The variance of $\widehat{a}_{m}$ is $\sigma_{\widehat{a}}^{2}=\sigma_{w}^{2} / N$.

\section{B. Iterative Phase Recovery Method}

The input to the algorithm is the sequence $\left\{\sqrt{I_{k}} e^{\mathrm{j} \hat{\phi}_{k}^{(i)}}\right\}_{0}^{N-1}$, where $\widehat{\phi}_{k}^{(i)}$ is the phase estimate at the $k$ th DFT index after the $i$ th iteration; $\widehat{\phi}_{k}^{(1)}$ is the initial phase. Under a low-noise condition, we have the Taylor-series approximation: $\sqrt{I_{k}}=\sqrt{\alpha_{k}+W_{k}}=$ $\sqrt{\alpha_{k}}+\left(W_{k} / 2 \sqrt{\alpha_{k}}\right)-\left(W_{k}^{2} / 8 \alpha_{k}^{3 / 2}\right)+\mathcal{O}\left(W_{k}^{3} / \alpha_{k}^{5 / 2}\right)$, where $\alpha_{k}=S_{k}\left|1+A_{k}\right|^{2}$ and $\mathcal{O}$ denotes the Landau symbol. The IDFT of $\left\{\sqrt{I_{k}} e^{\mathrm{j} \widehat{\phi}_{k}^{(i)}}\right\}_{0}^{N-1}$ is

$\widehat{d}_{m}^{(i)}=\frac{1}{N} \sum_{k=0}^{N-1}\left(\sqrt{\alpha_{k}}+\frac{W_{k}}{2 \sqrt{\alpha_{k}}}-\frac{W_{k}^{2}}{8 \alpha_{k}^{3 / 2}}\right) e^{\hat{\mathrm{\phi}}_{k}^{(i)}} e^{\mathrm{j} \frac{2 \pi}{N} k m}+$ h.o.t.,
$0<m<N-1$

where h.o.t. stands for higher-order terms. The sequence $\left\{\widehat{d}_{m}^{(i)}\right\}_{0}^{N-1}$, with the second-half part set to zero, yields $\left\{\widehat{a}_{m}^{(i)}\right\}_{0}^{N-1}$. Let the DFT of $\left\{\widehat{a}_{m}^{(i)}\right\}_{0}^{N-1}$ be denoted by $\widehat{g}_{k}^{(i)}$, the angle of which is used as the phase of $\sqrt{I_{k}}$ in the next iteration; i.e., $\widehat{\phi}_{k}^{(i+1)}=\angle \widehat{g}_{k}^{(i)}$. To proceed further with the calculations, consider the term $\mathcal{E}\left\{W_{k} e^{\mathrm{j} \hat{\phi}_{k}^{(i)}}\right\}=e^{\mathrm{j} \phi_{k}} \mathcal{E}\left\{W_{k} e^{\mathrm{j} \Delta \phi_{k}^{(i)}}\right\}$, where $\Delta \phi_{k}^{(i)}$ is the phase error in the $k$ th component at the $i$ th iteration. For large enough $i$, it is reasonable to write $\mathcal{E}\left\{W_{k} e^{\mathrm{j} \hat{\phi}_{k}^{(i)}}\right\} \approx e^{\mathrm{j} \phi_{k}} \mathcal{E}\left\{W_{k}\left(1-\mathrm{j} \Delta \phi_{k}^{(i)}-\left(\left(\Delta \phi_{k}^{(i)}\right)^{2} / 2\right)\right)\right\}$ in the small noise regime. This term is further scaled down by the factor $1 / 2 \sqrt{\alpha_{k}}$ [cf. (1)]. The magnitude of the product is proportional to $\sigma_{w}^{2} / \alpha_{k}$, which is small compared to $\alpha_{k}$. Therefore, its contribution to the bias is negligible. Similarly,

${ }^{1}$ The notation $\left\{X_{k}\right\}_{0}^{N-1}$ for sequences is used to indicate that $k$ takes values $0,1,2, \ldots, N-1$. 
the contribution of the term containing $W_{k}^{2}$ is also negligible. Under these approximations, we can write $a_{m}-\mathcal{E}\left\{\widehat{a}_{m}^{(i)}\right\} \approx$ $(1 / N) \sum_{k=0}^{N-1} \sqrt{\alpha_{k}} e^{\mathrm{j}(2 \pi / N) k m}\left(e^{\mathrm{j} \phi_{k}}-\mathcal{E}\left\{e^{\mathrm{j} \widehat{\phi}_{k}^{(i)}}\right\}\right)$. The expectation on the r.h.s. is rewritten as $\mathcal{E}\left\{e^{\mathrm{j} \hat{\phi}_{k}^{(i)}}\right\}=e^{\mathrm{j} \phi_{k}} \mathcal{E}\left\{e^{\mathrm{j} \Delta \phi_{k}^{(i)}}\right\} \approx$ $e^{\mathrm{j} \phi_{k}}\left\{1+\mathrm{j} \mathcal{E}\left\{\Delta \phi_{k}^{(i)}\right\}-\left(\mathcal{E}\left\{\left(\Delta \phi_{k}^{(i)}\right)^{2}\right\} / 2\right)\right\}$. Based on evidence from Monte Carlo trials, we found that the phase error has a negligible mean value, and hence its contribution to the above expectation is not significant. Denote $\sigma_{\Delta \phi_{k}^{(i)}}^{2}=\mathcal{E}\left\{\left(\Delta \phi_{k}^{(i)}\right)^{2}\right\}$, which is the variance of the zero-mean phase error after the $i$ th iteration. Therefore, we have that $\mathcal{E}\left\{e^{\mathrm{j} \hat{\phi}_{k}^{(i)}}\right\}=e^{\mathrm{j} \phi_{k}}\left\{1-\left(\sigma_{\Delta \phi_{k}^{(i)}}^{2} / 2\right)\right\}$. The bias can therefore be written as

$$
\left|a_{m}-\mathcal{E}\left\{\widehat{a}_{m}^{(i)}\right\}\right|=\frac{1}{2 N}\left|\sum_{k=0}^{N-1} \sigma_{\Delta \phi_{k}^{(i)}}^{2} \sqrt{\alpha_{k}} e^{\mathrm{j} \phi_{k}} e^{\mathrm{j} \frac{2 \pi}{N} k m}\right| .
$$

After convergence has been achieved, the above expression becomes independent of $i$. Therefore, the superscript $i$ can be dropped. We next compute an expression for the standard deviation. Recall that $\left\{\widehat{g}_{k}\right\}_{0}^{N-1}$ is the DFT of the causal part of $\left\{\widehat{d}_{k}\right\}_{0}^{N-1}$

$$
\begin{aligned}
\widehat{g}_{k} & =\sum_{m=0}^{N / 2-1} \widehat{d}_{m} e^{-\mathrm{j} \frac{2 \pi}{N} k m}=\frac{1}{N} \sum_{\ell=0}^{N-1} \sqrt{\alpha_{\ell}+W_{\ell}} e^{\mathrm{j} \widehat{\phi}_{\ell}} \beta_{\ell, k} \\
& =\frac{1}{N} \sum_{\ell=0}^{N-1} \sqrt{\alpha_{\ell}} e^{\mathrm{j} \widehat{\phi}_{\ell}} \beta_{\ell, k}+\frac{1}{N} \sum_{\ell=0}^{N-1} \frac{W_{\ell}}{2 \sqrt{\alpha_{\ell}}} e^{\mathrm{j} \widehat{\phi}_{\ell}} \beta_{\ell, k} .
\end{aligned}
$$

We write $e^{\widehat{\mathrm{j}}_{\ell}}=e^{\mathrm{j} \phi_{\ell}}\left\{1+\mathrm{j} \Delta \phi_{\ell}-\mathcal{O}\left(\Delta \phi_{\ell}^{2}\right)\right\}$, where $\widehat{\phi}_{\ell}=\phi_{\ell}+$ $\Delta \phi_{\ell}$. Similarly $\widehat{g}_{k} \approx\left|g_{k}\right| e^{\mathrm{j}_{\phi_{k}}} \approx g_{k}\left\{1+\mathrm{j} \Delta \phi_{k}\right\}$. From these expressions, we have that $\Delta \phi_{k} \approx\left(1 / 2 N g_{k}\right) \sum_{\ell=0}^{N-1}\left(W_{\ell} / \sqrt{\alpha_{\ell}}\right) e^{\mathrm{j} \phi_{\ell} \beta_{\ell, k}}$, where $\beta_{\ell, k}=\sum_{m=0}^{N / 2-1} e^{\mathrm{j}(2 \pi / N)(\ell-k) m}$ is deterministic. The variance of $\Delta \phi_{k}$ is given by $\sigma_{\Delta \phi_{k}}^{2}=\left(\sigma_{w}^{2} / 4 N^{2}\left|g_{k}\right|^{2}\right) \sum_{\ell=0}^{N-1}\left(\left|\beta_{\ell, k}\right|^{2} / \alpha_{\ell}\right)$. After convergence, in the noise-free case, we have $\left|g_{k}\right|^{2}=\alpha_{k}$. Therefore, (2) simplifies to

$$
\left|a_{m}-\mathcal{E}\left\{\widehat{a}_{m}\right\}\right|=\frac{\sigma_{w}^{2}}{8 N^{3}}\left|\sum_{k=0}^{N-1} \frac{1}{\sqrt{\alpha_{k}}} \sum_{\ell=0}^{N-1} \frac{\left|\beta_{\ell, k}\right|^{2}}{\alpha_{\ell}} e^{\mathrm{j} \phi_{k}} e^{\mathrm{j} \frac{2 \pi}{N} k m}\right| .
$$

Similarly, it can be shown that the variance is given by $\sigma_{\widehat{a}_{m}}^{2}=$ $\left(\sigma_{w}^{2} / 4 N^{2}\right) \sum_{k=0}^{N-1} \alpha_{k}$.

\section{Cepstral Technique}

The following analysis is in the same order as the operations described in Section III-C. Rewriting $I_{k}=\alpha_{k}\left(1+\left(W_{k} / \alpha_{k}\right)\right)$, and taking its logarithm, we get that

$$
\log I_{k}=\log S_{k}+\log \left|1+A_{k}\right|^{2}+\log \left(1+\frac{W_{k}}{\alpha_{k}}\right) .
$$

Recalling the Taylor-series expansion $\log (1+x)=x-\left(x^{2} / 2\right)+$ $\mathcal{O}\left(x^{3}\right)$ for $|x|<1$, we rewrite (4) as

$$
\log I_{k}-\log S_{k}=\log \left|1+A_{k}\right|^{2}+\underbrace{\frac{W_{k}}{\alpha_{k}}-\frac{W_{k}^{2}}{2 \alpha_{k}^{2}}}_{\eta_{k}}+\mathcal{O}\left(\frac{W_{k}^{3}}{\alpha_{k}^{3}}\right),
$$

the IDFT of which gives the estimated cepstrum

$$
\begin{aligned}
\widehat{c}_{m} & \approx \frac{1}{N} \sum_{k=0}^{k-1}\left(\log \left|1+A_{k}\right|^{2}+\eta_{k}\right) e^{\mathrm{j} \frac{2 \pi}{N} k m} \\
& =c_{m}+\frac{1}{N} \sum_{k=0}^{N} \eta_{k} e^{\mathrm{j} \frac{2 \pi}{N} k m}, \quad 0 \leq m \leq N-1
\end{aligned}
$$

where $\left\{c_{m}\right\}_{0}^{N-1}$ is the cepstrum in the absence of noise. The DFT of the causal part of $\left\{\widehat{c}_{m}\right\}_{0}^{N-1}$ is

$$
\widehat{C}_{k}^{+}=C_{k}^{+}+\frac{1}{N} \sum_{\ell=0}^{N-1} \eta_{\ell} \underbrace{\sum_{m=0}^{N / 2-1} e^{j} \frac{2 \pi}{N}(\ell-k) m}_{\beta_{\ell, k}}+\text { h.o.t. }
$$

The step (4) of the technique (cf. Section III-C) yields

$$
\begin{aligned}
\widehat{A}_{k} & =\exp \left(\widehat{C}_{k}^{+}\right)-1 \\
& =\exp \left(C_{k}^{+}\right)\left(1+\frac{1}{N} \sum_{\ell=0}^{N-1} \beta_{\ell, k} \eta_{\ell}\right)-1+\text { h.o.t. } \\
& =A_{k}+\exp \left(C_{k}^{+}\right)\left(\frac{1}{N} \sum_{\ell=0}^{N-1} \beta_{\ell, k} \eta_{\ell}\right)+\text { h.o.t. }
\end{aligned}
$$

The IDFT of the sequence $\left\{\widehat{A}_{k}\right\}_{0}^{N-1}$ gives the scattering function

$$
\begin{array}{r}
\widehat{a}_{m}=a_{m}+\frac{1}{N^{2}} \sum_{\ell=0}^{N-1} \eta_{\ell} \underbrace{}_{\gamma_{\ell, m} \sum_{k=0}^{N-1} \exp \left(C_{k}^{+}\right) \beta_{\ell, k} e^{\mathrm{j} \frac{2 \pi}{N} k m}}+\text { h.o.t., } \\
\quad 0 \leq m \leq \frac{N}{2}-1 .
\end{array}
$$

It follows from (9) that $\mathcal{E}\left\{\widehat{a}_{m}\right\} \approx a_{m}-\left(\sigma_{w}^{2} / 2 N^{2}\right) \sum_{\ell=0}^{N-1}\left(\gamma_{\ell, m} / \alpha_{\ell}^{2}\right)$. The variance of $\widehat{a}_{m}$ is given by

$$
\begin{array}{r}
\sigma_{\widehat{a}_{m}}^{2}=\frac{1}{N^{4}}\left(\sum_{\ell=0}^{N-1}\left|\gamma_{\ell, m}\right|^{2}\left\{\frac{\sigma_{w}^{2}}{\alpha_{\ell}^{2}}+\frac{\sigma_{w}^{4}}{4 \alpha_{\ell}^{4}}\right\}+\sigma_{w}^{4}\left|\sum_{\ell=0}^{N-1} \frac{\gamma_{\ell, m}}{2 \alpha_{\ell}^{2}}\right|^{2}\right. \\
\left.+\sigma_{w}^{2} \operatorname{Re}\left\{\left(\sum_{\ell=0}^{N-1} \frac{\gamma_{\ell, m}}{2 \alpha_{\ell}^{2}}\right)\left(\sum_{\ell=0}^{N-1} \frac{\gamma_{\ell, m}^{*}}{\alpha_{\ell}^{2}}\right)\right\}\right) .
\end{array}
$$

\section{Monte Carlo Analyses}

Deconvolution of the source spectrum is implicit in the cepstral technique, but this is not the case with the Fourier and iterative methods. Therefore, in order to make a fair comparison, we set the source spectrum to unity within the measurement bandwidth. We synthesize a scattering function (cf. Fig. 2), motivated by measurements on standard phantoms such as fiber optic cables [19], microscope glass slides, multilayer silica-glass phantoms [15], [18], etc. We generate 500 realizations of pseudorandom white Gaussian noise and compute the scattering function estimates by using the three techniques. For the iterative method, we employed the log-Hilbert-transform-based initialization proposed in [10] to speed up convergence. Typically, the number of iterations needed would be about 10 [10], but we iterate 100 times to make sure that the best results are obtained. The experiment is repeated for different values of SNR; corresponding to each value, the biases and variances are computed.

The biases of the estimators corresponding to $\mathrm{SNR}=25 \mathrm{~dB}$ are shown in Fig. 3. The bias in the Fourier approach is the autocorrelation term. The result of the simulation matches with the theoretical results for small lags and at the peaks. The bias at larger lags and in the valleys is dominated by noise. The theoretical bias for the iterative method appears to be reasonably accurate. Although the theoretical bias of the cepstral technique is small compared to the Monte Carlo value, in practice, the noise floor places a limit. The Monte Carlo bias profiles of the iterative and cepstral techniques are nearly identical.

The variances of the estimates for SNR $=25 \mathrm{~dB}$ are shown in Fig. 4 We see that the simulation results are in agreement with the theoretical 


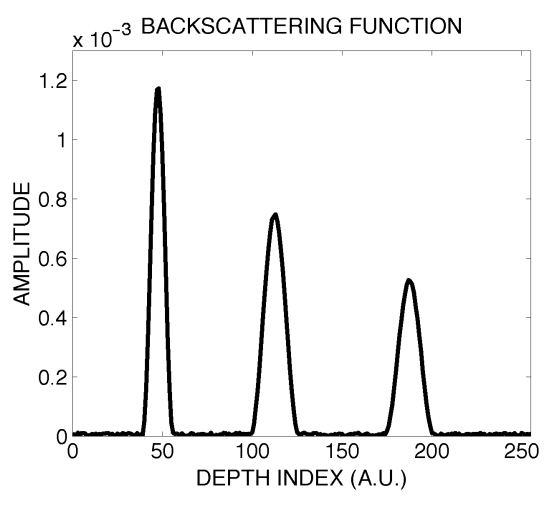

Fig. 2. The scattering function used for Monte-Carlo analysis (cf. Section V). A.U. stands for arbitrary units.
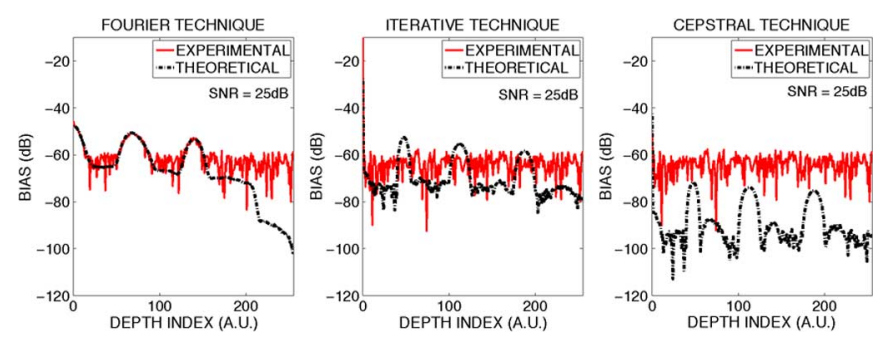

Fig. 3. Biases $(\mathrm{SNR}=25 \mathrm{~dB})$ versus the axial depth index.
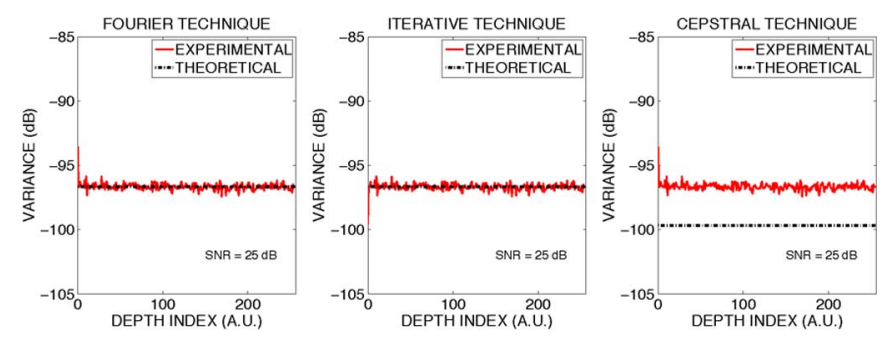

Fig. 4. Variances $(\mathrm{SNR}=25 \mathrm{~dB})$ versus the axial depth index.
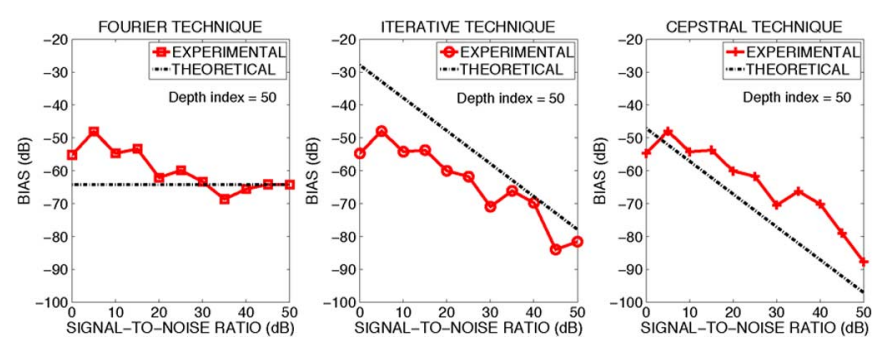

Fig. 5. Biases at the first peak (index $=50$ ) versus SNR.

predictions for the Fourier and iterative techniques. For the cepstral technique, there is a small discrepancy between the theoretical prediction and the experimental value. From these plots, we infer that the calculations are fairly accurate. Also, note that the three techniques have nearly identical Monte Carlo variance profiles.

Another important aspect is the SNR dependence of the bias and variance. We show in Figs. 5 and 6 the biases and variances of the estimators versus the SNR, corresponding to a point at the peak of the first interface (index $=50$ ) shown in Fig. 2. The bias of the Fourier method saturates and matches with the theoretical prediction, whereas the biases of the iterative and cepstral techniques decrease with increase
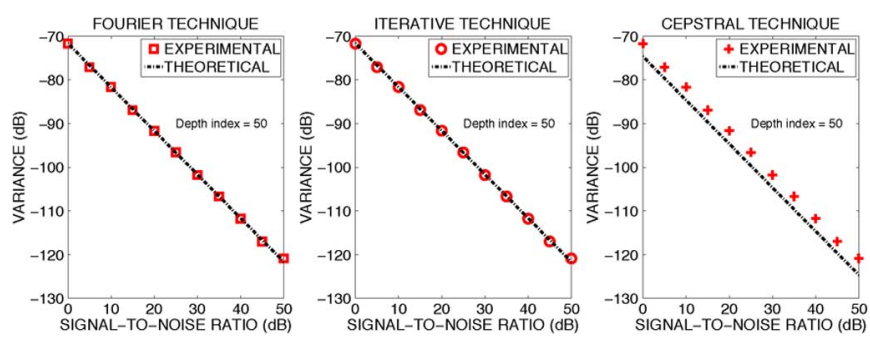

Fig. 6. Variances at the first peak (index $=50$ ) versus SNR.
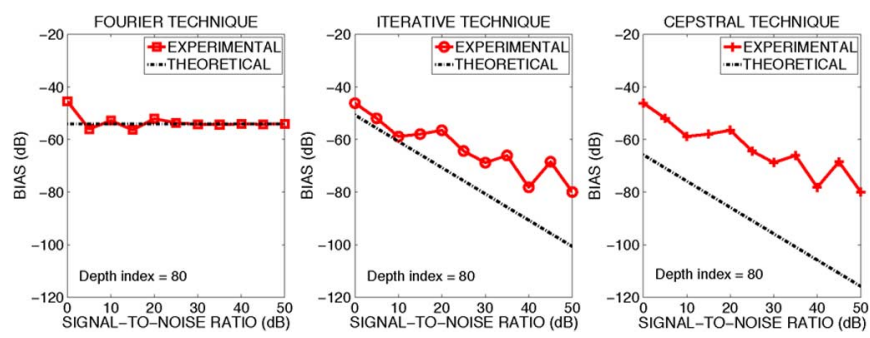

Fig. 7. Biases versus SNR at index $=80$.
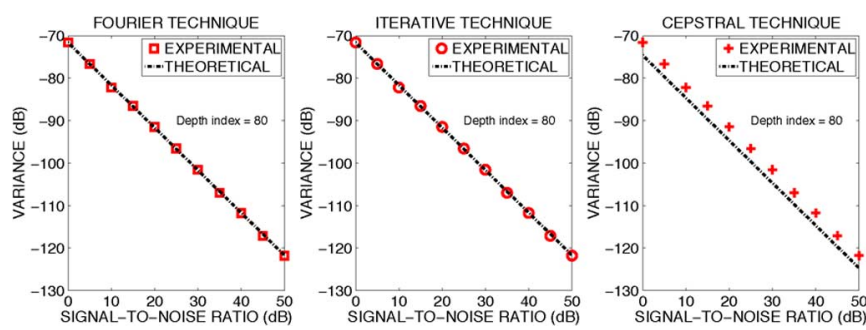

Fig. 8. Variances versus SNR at index $=80$.

in SNR. By comparing the Monte Carlo variances across techniques, we see that they are almost identical and that there is a good match between the theoretical and simulation results. The variances also have a linear dependence on the SNR.

In Figs. 7 and 8, we show another set of results corresponding to the valley between the first and second peaks in the scattering function (index $=80$ ). The bias of the Fourier approach saturates because the autocorrelation has a peak around this point and thus limits the achievable accuracy. For the other two techniques, the bias shows a decreasing trend. At this location, the theoretical predictions for the iterative and cepstral techniques are not expected to match with the simulation results mainly because of the local absence of signal (i.e., no interface), and therefore the assumption of high SNR, under which the expressions are derived, does not hold. There is some discrepancy between the theoretical and experimental biases for the iterative and cepstral techniques suggesting that the truncated Taylor-series calculations may be suitable for determining the variance, but not sufficiently accurate for determining the bias. The general observation is that the three techniques have nearly identical variances, but the iterative and cepstral techniques give rise to lesser bias than the Fourier method. Such observations were also found to hold for a different choice of the scattering function.

\section{CONCLUSION}

We have compared the noise sensitivities of three state-of-the-art reconstruction techniques for FDOCT. We have shown that the Fourier method has a higher bias than the iterative and the cepstral techniques. The experimental results are in agreement with the theoretical values except for a small discrepancy in the bias for the iterative and cepstral 
techniques. The cepstral technique, although nonlinear, yields estimators with a variance that equals that of the Fourier and iterative methods. Therefore, in switching from the Fourier approach to the cepstral technique, statistical efficiency is not lost; in fact, there is a gain in the reconstruction accuracy. The price paid for this migration is only a small increase in algorithm complexity.

\section{REFERENCES}

[1] A. F. Fercher, C. K. Hitzenberger, G. Kamp, and S. Y. El-Zaiat, "Measurement of intraocular distances by backscattering spectral interferometry," Opt. Commun., vol. 117, pp. 43-48, 1995.

[2] J. G. Fujimoto, M. E. Brezinski, G. J. Tearney, S. A. Boppart, B. E. Bouma, M. R. Hee, J. F. Southern, and E. A. Swanson, "Optical biopsy and imaging using optical coherence tomography," Nat. Med., vol. 1, pp. 970-972, 1995.

[3] J. A. Izatt, M. D. Kulkarni, K. Kobayashi, M. V. Sivak, J. K. Barton, and A. J. Welsh, "Optical coherence tomography for biodiagnostics," Opt. Photon. News, vol. 8, pp. 41-47, 1997.

[4] A. F. Fercher, "Optical coherence tomography," J. Biomed. Opt., vol. 1, pp. $157-173,1996$

[5] J. M. Schmitt, "Optical coherence tomography (OCT): A review," IEEE J. Sel. Topics Quantum Electron., vol. 5, no. 4, pp. 1205-1215, Jul./ Aug. 1999.

[6] P. H. Tomlins and R. K. Wang, "Theory, developments and applications of optical coherence tomography," J. Phys. D: Appl. Phys., vol. 38, no. 15, pp. 2519-2535, 2005.

[7] R. K. Wang and S. Hurst, "Mapping of cerebro-vascular blood perfusion in mice with skin and skull intact by optical micro-angiography at $1.3 \mu \mathrm{m}$ wavelength," Opt. Exp., vol. 15, no. 18, pp. 11402-11412, 2007.

[8] R. K. Wang, S. L. Jacques, Z. Ma, S. Hurst, S. R. Hanson, and A. Gruber, "Three dimensional optical angiography," Opt. Exp., vol. 15, no. 7, pp. 4083-4097, 2007.
[9] R. A. Leitgeb, "Optical coherence tomography-High-resolution imaging of structure and function," in Proc. 29th Int. Conf. IEEE EMBS, Aug. 2007, pp. 530-532.

[10] A. Ozcan, M. J. F. Digonnet, and G. S. Kino, "Minimum-phase-function-based processing in frequency-domain optical-coherence tomography systems," J. Opt. Soc. Amer. A, vol. 23, no. 7, pp. 1669-1677, Jul. 2006.

[11] C. S. Seelamantula, M. L. Villiger, R. A. Leitgeb, and M. Unser, "Exact and efficient signal reconstruction in frequency-domain optical-coherence tomography," J. Opt. Soc. Amer. A, vol. 25, no. 7, pp. 1762-1771, Jul. 2008.

[12] Handbook of Optical Coherence Tomography, B. E. Bouma and G. J. Tearney, Eds. New York: Marcel Dekker, 2002.

[13] T. F. Quatieri, Jr. and A. V. Oppenheim, "Iterative techniques for minimum phase signal reconstruction from phase or magnitude," IEEE Trans. Acoust., Speech, Signal Process., vol. ASSP-29, no. 6 , pp. 1187-1193, Dec. 1981.

[14] J. R. Fienup, "Phase retrieval algorithms: A comparison," Appl. Opt., vol. 21, pp. 2758-2769, 1982.

[15] R. A. Leitgeb, C. K. Hitzenberger, and A. F. Fercher, "Performance of Fourier-domain vs. time-domain optical-coherence tomography," Opt. Exp., vol. 11, no. 8, pp. 889-894, Apr. 2003.

[16] Z. Yaqoob, D. Psaltis, M. S. Feld, and C. Yang, "Optical phase conjugation for turbidity suppression in biological samples," Nature Photon. vol. 2, pp. 110-115, Febuary 2008.

[17] T. S. Ralston, I. Atkinson, F. Kamalabadi, and S. A. Boppart, "Multidimensional denoising of real-time OCT imaging data," in Proc. 31st IEEE Int. Conf. Acoust., Speech, Signal Process., May 2006, vol. 2, pp. $1148-1151$.

[18] P. H. Tomlins and R. K. Wang, "Matrix approach to quantitative refractive index analysis by Fourier domain optical coherence tomography," J. Opt. Soc. Amer. A, vol. 23, no. 8, pp. 1897-1907, 2006.

[19] J. C. Jasapara, S. Wielandy, and A. D. Yablon, "FDOCT-A new platform for measurement of standard and microstructured fibre dimensions," IEE Proc. Optoelectron., vol. 153, no. 5, pp. 229-234, Oct. 2006. 